\title{
Overlapping Signal Separation Method Using Superresolution Technique Based on Experimental Echo Shape
}

\author{
Jihad Al-Oudatallah, ${ }^{1}$ Fariz Abboud, ${ }^{1}$ Mazen Khoury, ${ }^{2}$ and Hassan Ibrahim ${ }^{2}$ \\ ${ }^{1}$ Department of Electronics and Communications, Damascus University, Damascus, Syria \\ ${ }^{2}$ Higher Institute for Applied Science and Technology, Damascus, Syria \\ Correspondence should be addressed to Jihad Al-Oudatallah; j_aloudh@hotmail.com
}

Received 30 January 2017; Revised 5 April 2017; Accepted 18 April 2017; Published 11 June 2017

Academic Editor: Jorge P. Arenas

Copyright (C) 2017 Jihad Al-Oudatallah et al. This is an open access article distributed under the Creative Commons Attribution License, which permits unrestricted use, distribution, and reproduction in any medium, provided the original work is properly cited.

Overlapping signals separation is a difficult problem, where time windowing is unable to separate signals overlapping in time and frequency domain filtering is unable to separate signals with overlapping spectra. In this work, a simulation under MATLAB is implemented to illustrate the concept of overlapping signals. We propose an approach for resolving overlapping signals based on Fourier transform and inverse Fourier transform. The proposed approach is tested under MATLAB, and the simulation results validate the effectiveness and the accuracy of the proposed approach. The approach is developed using Gerchberg superresolution technique to cope with signals with low signal-to-noise ratio. For practical work, an echo shape determination is required to apply the proposed technique. The experimental results show accurate localization of multiple targets.

\section{Introduction}

In measurement systems based on pulse echo techniques, some problems may occur when received signal contains many overlapping reflected echoes due to many factors: multiple targets, structure of the propagation media that may be consisting of several layers; and the complex physical properties of the propagation path. Therefore, it is important to find an approach for overlapping echoes separation encountered in a wide range of applications such as military and biomedical applications, [1-5].

Overlapping problem occurs in both time and frequency domains. Time overlapping signals are encountered when time delay between echoes is shorter than the emitted signal's duration. Overlapping is due to a combination of layer thickness or distance between reflectors $[1,5]$.

A difficulty arises in separating the composite signal, when two or more of the individual component signals have spectra that are the same or similar. This is also a well-known difficulty in musical sound separation when the harmonics of two or more pitched instruments have similar frequencies [6].
The frequency-phase relationship of the individual signals is incoherent causing constructive and destructive interference, resulting in irregular characteristics of the spectrum of the composite signal rather than the individual signals [1]. So the relative phase of overlapping signals is found to influence the precision of frequency determination and plays a critical role in the observed amplitude of the combined signal; thus the amplitude and the phase of individual signals become unobservable $[6,7]$.

So it is a challenging problem in signal processing to obtain the spectrum and the time localization of the individual component signals, as they overlap in both time and frequency domains, because it is difficult for traditional methods like spectrum method and time-frequency method to separate overlapping signals. Time domain windowing is unable to separate signals overlapping in time. Frequency domain filtering is unable to separate spectrally overlapped signals due to the similarity between the spectra of the signals.

Previous studies related to signal separation have emerged in recent years. Several different approaches such as short-time Fourier transform, Wigner-Ville distribution, discrete wavelet transform, discrete cosine transform, and 
chirplet transform, and Fractional Fourier transform have been proposed to cope with the overlapping problem $[1,8]$.

In this paper, we propose a method for resolving overlapping signals based on Fourier transform and inverse Fourier transform. Proposed method performance for noisy signals is improved using Gerchberg superresolution technique.

To apply the proposed method in practical application (directed acoustic transmission-reception system in our case), it is necessary to obtain the echo shape derived from experiments, so Hilbert transform is used to extract the received signal envelop, and the extracted envelop is used to modulate the transmitted signal.

This paper is organized as follows: Section 2 introduces the concept of overlapping signals and illustrates the problem encountered by a simulation under MATLAB. In Section 3, the proposed technique is introduced and illustrated by simulation results. Section 4 presents experimental echo shape determination by extracting the received signal envelop using Hilbert transform, where the transmitted signal is modulated by the extracted envelop. Section 5 shows experimental examples results of applying the proposed technique to received signal, using acoustic transmission-reception system, and multiple targets. Finally, conclusion is drawn in Section 6.

\section{Concept of Overlapping Signals}

The concept of overlapping signals is illustrated by a simulation implemented in MATLAB. The composite signal is a summation of a number of individual component signals (three overlapping echoes); the individual component signals have the same frequency $(2 \mathrm{kHz})$, different magnitudes, and different time delays as shown in Figure 1. The summation of overlapping signals is shown in Figure 2. Figure 3 shows the spectra of the three individual component signals after Fourier transformation, and Figure 4 shows the spectrum of the composite signal after Fourier transformation.

In all simulations, the following parameters will be used: the sampling frequency $f_{\mathrm{s}}=100 \mathrm{kHz}$ and the acquisition time $T=1 \mathrm{sec}$, so the number of samples $N=100000$, discrete Fourier transform (DFT) computed with a 100000point FFT, and the frequency increment $d f=1 \mathrm{~Hz}$.

As illustrated in Figure 4, frequency domain analysis of time overlapping signals using Fourier transformation does not produce the spectrum of the individual component signals. The individual component signals have regular spectra and main lobe centred at $(2 \mathrm{kHz})$, but the spectrum of the composite signal has distortions and irregular characteristics.

\section{Proposed Method}

3.1. Theoretical Background. Assuming that the transmitted signal is $x(t)$,

$$
x(t)=w(t) \cdot \sin 2 \pi f_{0} t
$$

where $w(t)$ is the function shape of the transmitted pulse and $f_{0}$ is the transmission frequency. The received signal $y(t)$

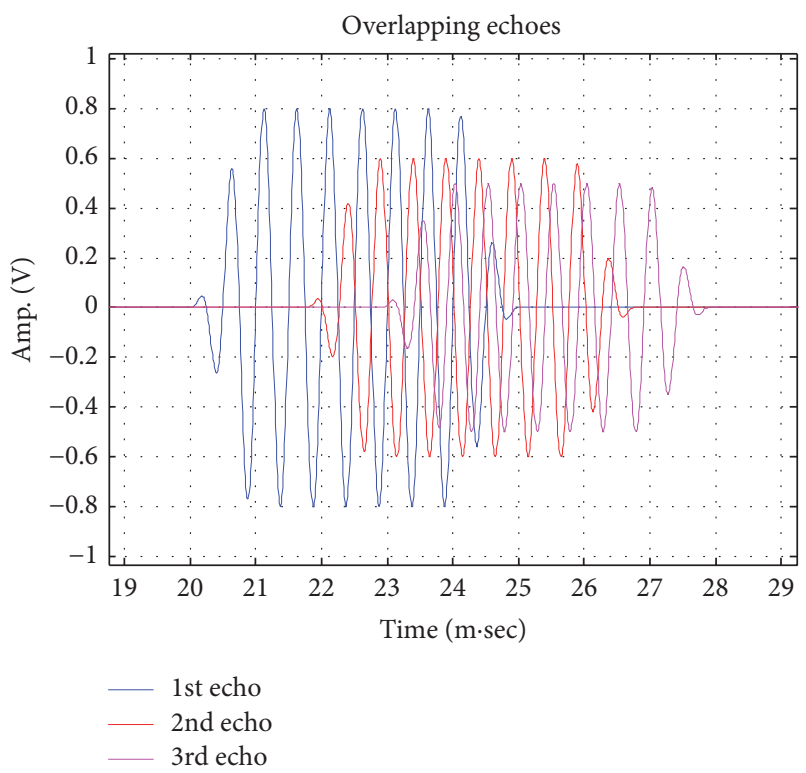

FIGURE 1: Three overlapping echoes.

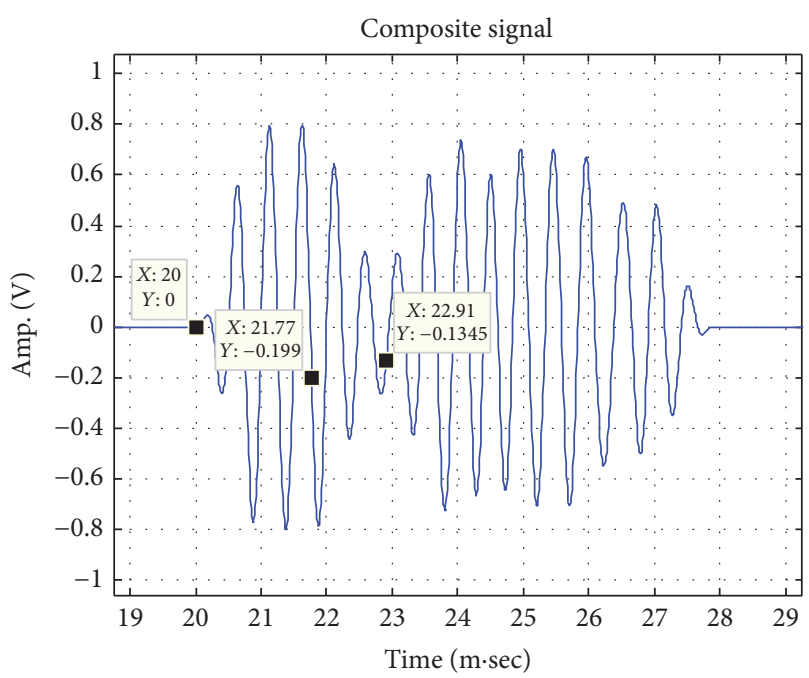

FIGURE 2: Composite signal.

could, for instance, be several $(n)$ scaled and time delayed copies of a known signal $x(t)$ :

$$
y(t)=\sum_{k=1}^{n} a_{k} x\left(t-\tau_{k}\right),
$$

where $a_{k}$ is the amplitude of the shape of the $k$ th echo pulse and $\tau_{k}$ is its time delay. By taking the Fourier Transform of (2), we get

$$
Y(f)=\sum_{k=1}^{n} a_{k} X(f) e^{-j 2 \pi f_{0} \tau_{k}}
$$




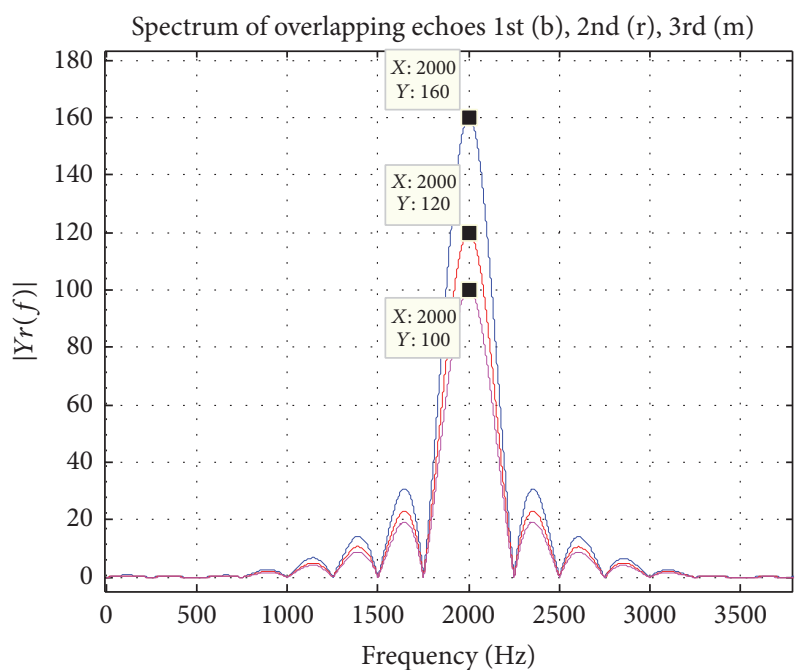

FIGURE 3: Spectra of the individual signals.

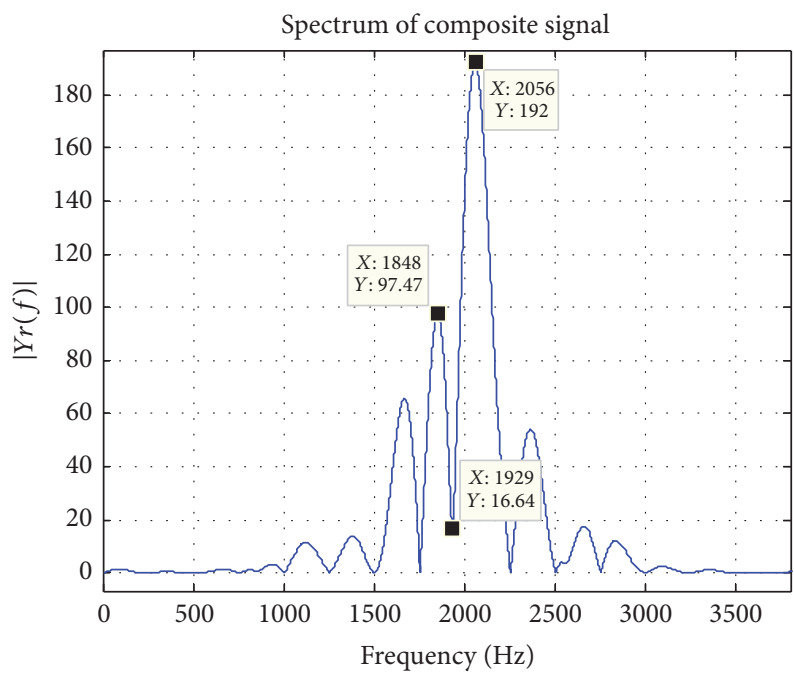

Figure 4: Spectrum of the composite signal.

where $X(f)$ is the Fourier Transform of (1). We define the transform function $H(f)$ in the frequency domain by

$$
H(f)=\frac{Y(f)}{X(f)}=\sum_{k=1}^{n} a_{k} e^{-j 2 \pi f_{0} \tau_{k}} .
$$

By taking the Inverse Fourier Transform of (4), we get

$$
h(t)=\sum_{k=1}^{n} a_{k} \delta\left(t-\tau_{k}\right) .
$$

Equation (5) shows that the resulting transform function in the time domain is several $(n)$ pulses $\delta(t)$ with amplitude $a_{k}$ and time delay $\tau_{k}$.

By applying this method on signal illustrated in Figure 2, where $y(t)$ is the composite signal, we get transform functions in frequency domain $H(f)$ and time domain $h(t)$ as shown in Figures 5 and 6.

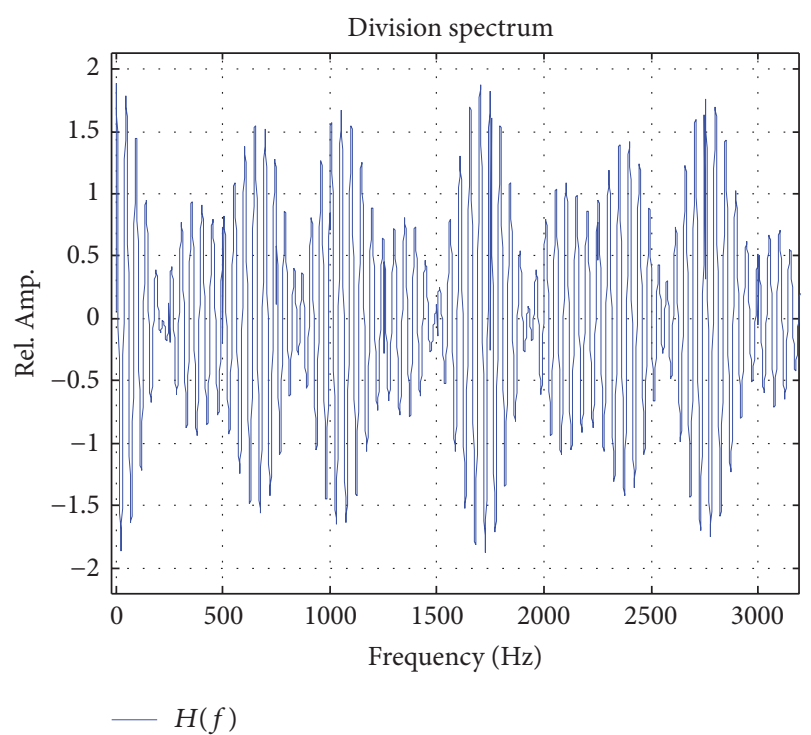

FIgURE 5: Transform function $H(f)$ in frequency domain.

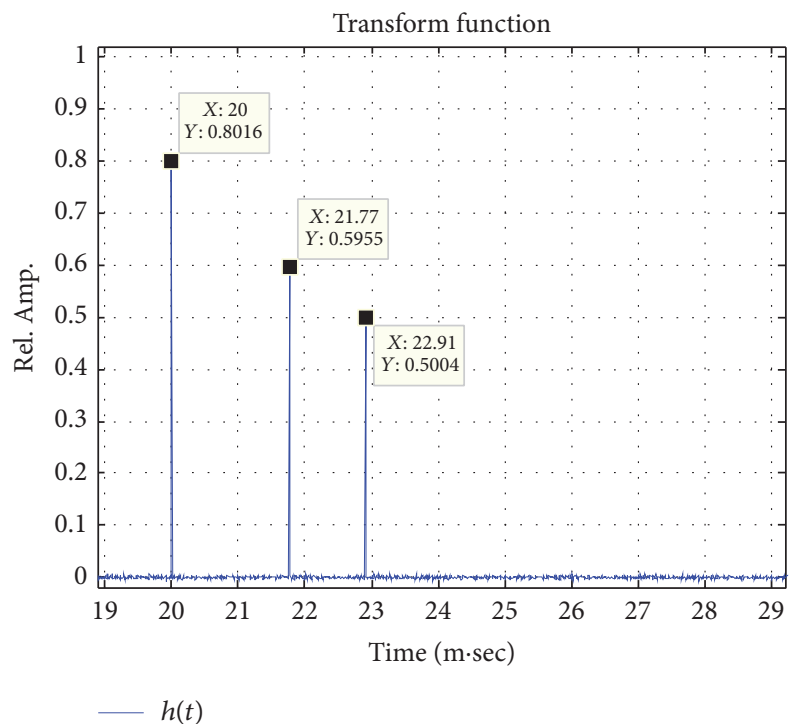

FIgURE 6: Transform function $h(t)$ in the time domain.

Note that time delays and amplitudes of echoes, obtained in Figure 6, are in agreement with those given in Figure 1.

3.2. Effect of Signal-to-Noise Ratio (SNR). In practical application, signals could not be as the simulated transmitted and received signals due to the noise, frequency shift, and system response.

Unfortunately, the proposed method is very sensitive to noise, which may cause a catastrophic effect on results; to illustrate this point, a white noise with signal-to-noise ratio $(\mathrm{SNR}=20)$ will be added to the composite signal shown in Figure 2, so the noisy composite signal becomes as shown in Figure 7. Transform functions $H(f)$ and $h(t)$ in frequency and time domain are shown in Figures 8 and 9, respectively. 


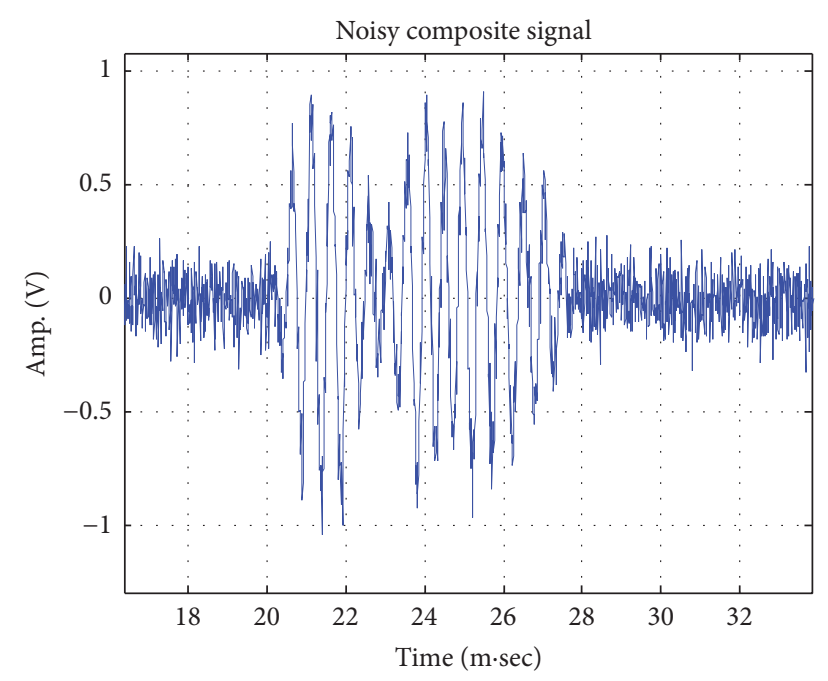

FIGURE 7: The composite signal corrupted by additive noise.

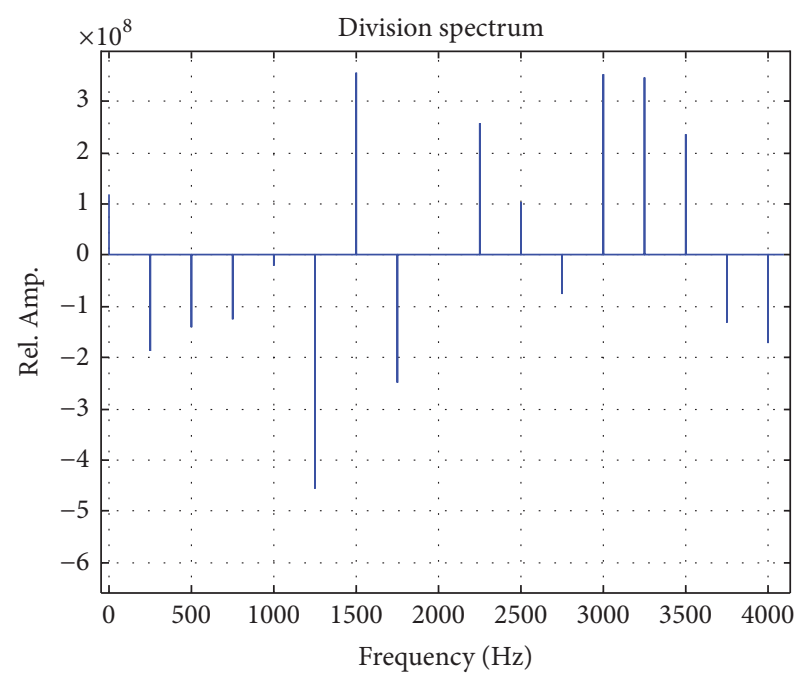

FIgURE 8: Transform function $H(f)$ of composite signal corrupted by additive noise in frequency domain.

Narrow pulses with high amplitude shown in Figure 8 are caused by dividing by zeros (of $X(f)$ ), resulting in periodic sinusoidal signals in all the time domain, as shown in Figure 9; these signals cover pulses at time delays $\tau_{k}$ of our interest.

To cope with problem, (4) could be developed as the following form:

$$
H(f)=\frac{Y(f) \cdot X^{*}(f)}{X(f) \cdot X^{*}(f)+\gamma}=\frac{F\left(R_{y x}(\tau)\right)}{F\left(R_{x x}(\tau)\right)+\gamma},
$$

where $\gamma$ is a small real value related to SNR, representing the ratio of the power spectral density of the noise and that of the signal [9], $X^{*}(f)$ is the complex conjugate of $X(f)$, $F\left(R_{y x}(\tau)\right)$, and $F\left(R_{x x}(\tau)\right)$ are Fourier transforms of crosscorrelation and autocorrelation functions, respectively [10]. This technique used in (6) is similar to the Wiener Filter used in image processing [9], and it is powerful in measurement

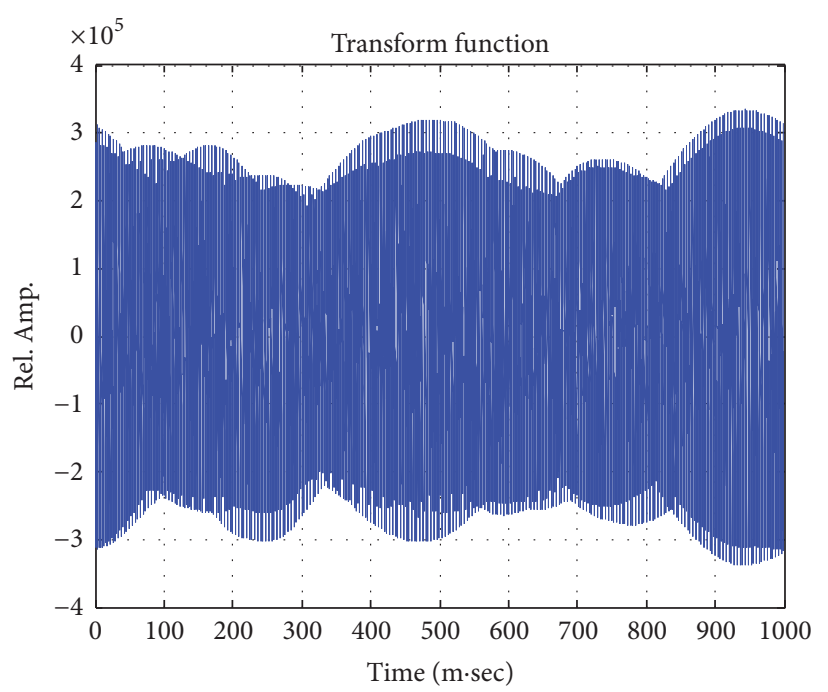

FIGURE 9: Transform function $h(t)$ of composite signal corrupted by additive noise in time domain.

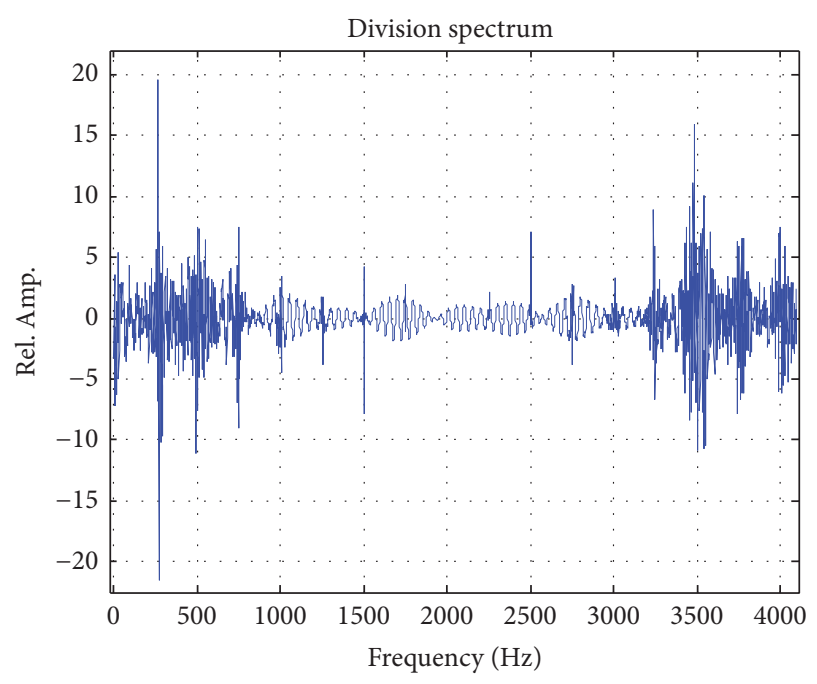

FIGURE 10: Transform function $H(f)$ according to (6) with $\gamma=0.05$.

conditions with low $\mathrm{S} / \mathrm{N}$ ratio, where the autocorrelation function contains the same information about the signal, and, by using the cross-correlation function, it is possible to obtain an increase in the $\mathrm{S} / \mathrm{N}$ ratio (the Wiener-Khintchine theorem) $[11,12]$.

Figure 10 shows the transform function $H(f)$ of previous example according to (6) with $\gamma=0.05$.

As shown in Figure 10, the technique in used in (6) has reduced the noise effect (the effect of dividing by zero), but this is not enough to recognize pulses of interest in transform function $h(t)$, so an improvement based on super resolution technique is required.

3.3. Superresolution Technique. The proposed method performance is improved using Gerchberg superresolution technique [13], as illustrated in the following steps: 
(1) The received signal is windowed by a time window $\left[\begin{array}{ll}t 1 & t 2\end{array}\right]$, with zero padding outside the window, where $t 1$ and $t 2$ are limits of time window of interest; this is done by detection algorithm or by dividing time domain of the received signal into predefined timelength frames.

(2) Taking the Fourier transform of the signal obtained in step $1,(6)$ is used with a suitable predefined value for $\gamma$; then we get $H_{p}(f)=H(f)$.

(3) Transform function $H(f)$ obtained in step 2 is filtered by time band filter [ $t 1 t 2]$, where $t 1$ and $t 2$ are limits of the same time window in step $1, H_{c}(f)$ is the filtered transform function of $H(f)$, and $H_{w}(f)$ is defined as the following:

$$
H_{w}(f)= \begin{cases}H_{p}(f) ; & \text { for } f_{1}<f<f_{2} \\ H_{c}(f) ; & \text { otherwise }\end{cases}
$$

where $f_{1}$ and $f_{2}$ are frequency band limits of interest (around the transmission frequency $f_{1}<f_{0}<f_{2}$ ).

(4) Taking the inverse Fourier transform of $H_{w}(f)$, obtained transform function $h(t)$ is windowed by the same time window [ $t 1 t 2]$, but, to compensate the filter delay used in step 3 , time window is shifted by the same filter delay and then $h_{w}(t)$ is obtained.

(5) Taking Fourier transform of $h_{w}(t)$ to get $H_{c}(f)$ and $H_{p}(f)=H_{w}(f),(7)$ is used to get a new $H_{w}(f)$.

(6) $H_{m}(f)$ is filtered $H_{w}(f)$ by time band filter [ $\left.t 1 t 2\right]$, and $h_{m}(t)$ is the inverse Fourier transform of $H_{m}(f)$.

(7) A predefined number of iterations are used to get the best resolution for $h_{m}(t)$ by successive steps: taking the Fourier transform of the windowed $h_{m}(t)$ with filter delay compensation to get $H_{c}(f)$ and $H_{p}(f)=$ $H_{m}(f),(7)$ is used to get a new $H_{w}(f)$, filtering $H_{w}(f)$ by time band filter [ $t 112]$ to get new $H_{m}(f)$, taking inverse Fourier transform of $H_{m}(f)$ to get new $h_{m}(t)$.

Modified transform functions $\mathrm{Hm}(f), h m(t)$ (red curve), and its absolute value (blue curve) of the previous example, in frequency and time domain, of the third-order iteration are shown in Figures 11 and 12, respectively.

Time delays obtained in Figure 12 are in agreement with those obtained in Figure 6 and given in Figure 1.

In practical application, the problem encountered is to get the reference transmitted signal that really propagates in the medium, where the received signal is considered to be several scaled and time delayed copies of it. So we have to construct heuristically the reference transmitted signal that has the same transmission frequency $f_{0}$ but modulated by shape function (pulse envelope); it is supposed that echoes have the same shape but with scaled amplitude.

In the following paragraph, we propose an approach to extracting the pulse envelope from the received signal using Hilbert transform.

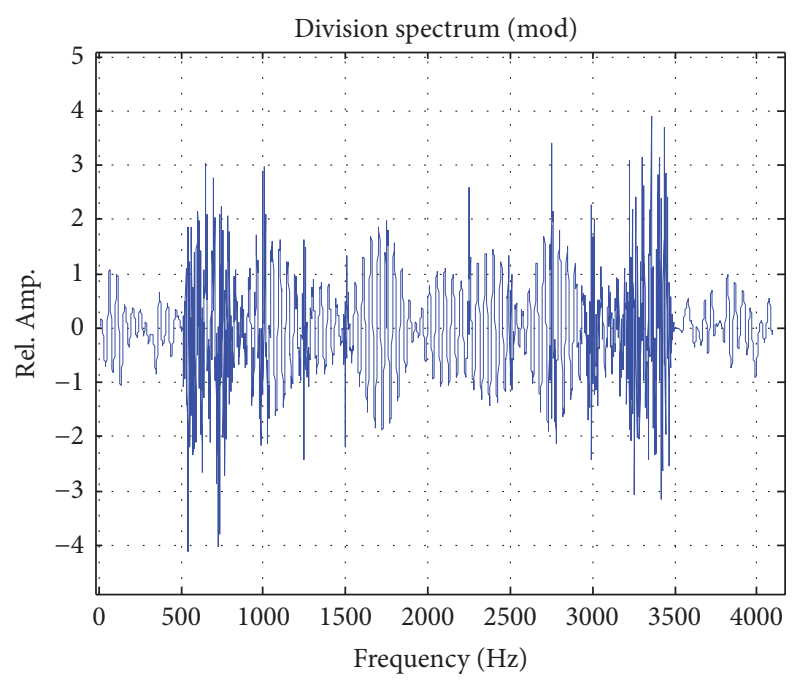

FIgURE 11: Modified transform function $H m(f)$ in frequency domain.

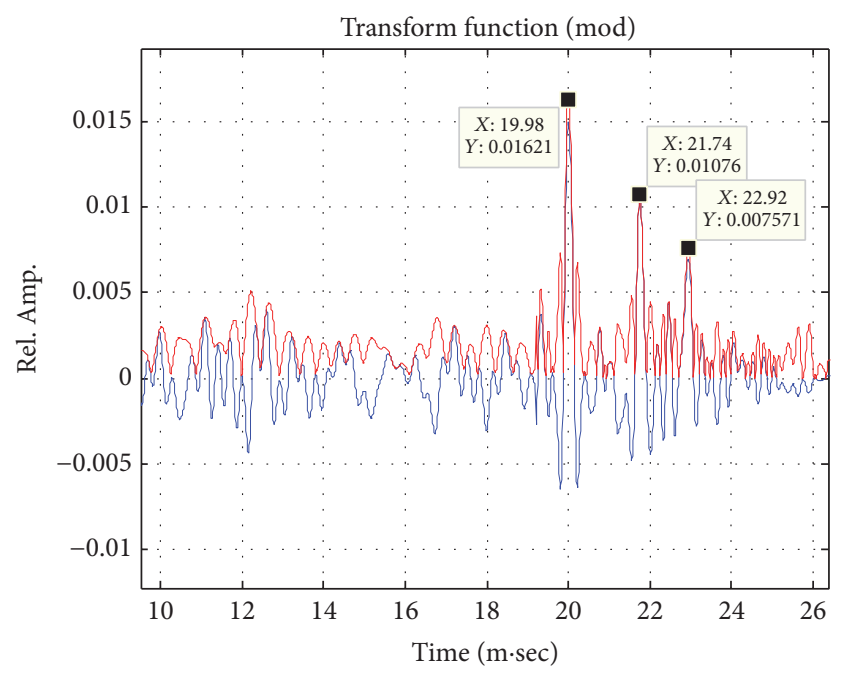

FIgURE 12: Modified transform function $h m(t)$ in time domain.

\section{Experimental Envelope Detection}

The Hilbert transform facilitates the formation of the analytic signal $x_{\mathrm{a}}(t)$. The analytic signal is useful in the area of communications, particularly in pass band signal processing.

Figure 13 shows the schematic representation of experimental setup used for envelope detection.

This experimental setup is used in a closed and empty Basketball Hall that is considered a nearly anechoic environment, where microphone and loudspeaker are located about $2 \mathrm{~m}$ above the ground, and the distance from the microphone or the loudspeaker to the closest object is greater than $5 \mathrm{~m}$.

First, we generate 10 periods of mathematical sine wave at $\left(f_{0}=2000 \mathrm{~Hz}\right)$ frequency with a rectangular envelope as a transmission pulse (pulse width $=5 \mathrm{~m} \cdot \mathrm{sec}$ ). Using a laptop, this pulse is transmitted through a sound card, an acoustic power amplifier, and a loudspeaker. Using a microphone, the pulse is received directly and acquired through the sound 


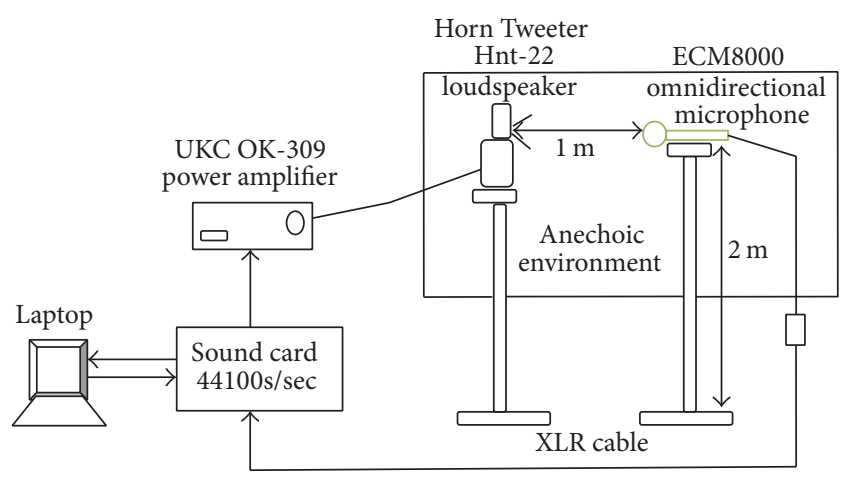

FIGURE 13: Schematic representation of experimental setup used for envelope detection.

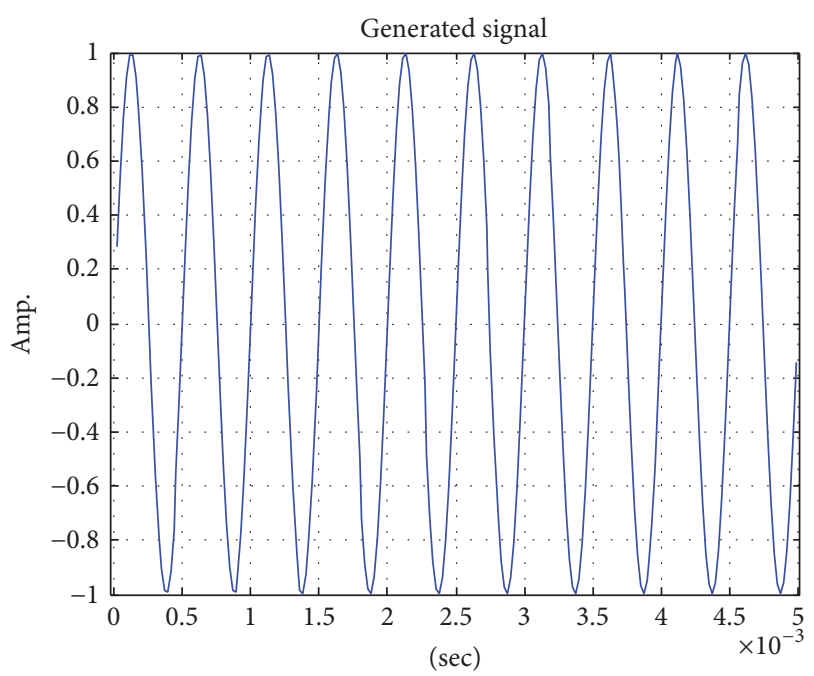

FIgUre 14: Mathematical generated signal.

card; the received signal $x(t)$ is processed to obtain its analytic signal $x_{\mathrm{a}}(t)$, that is,

$$
x_{\mathrm{a}}(t)=A(t) e^{j \Phi(t)}=x(t)+j H(x(t)),
$$

where $A(t)$ is the instantaneous amplitude and $H(x(t))$ is the Hilbert Transform of $x(t)$, which is given by $[14,15]$

$$
H(x(t))=\frac{1}{\pi} \int_{-\infty}^{\infty} \frac{x(\tau)}{t-\tau} d \tau .
$$

Figure 14 shows the mathematical generated signal. Figure 15 shows the experimental reference signal and its extracted envelope using Hilbert transform, implemented under MATLAB. This envelope derived from experiments (received signal shifted to time 0 ), supposed to be similar to echo shape, is used to modulate mathematical sine wave at $f_{0}$ frequency to be the reference transmitted signal. This model of transmitted signal and reflected echoes takes into account the response of the transmitter and receiver sensors, the number of cycles of the excitation pulse, and the propagation medium characteristics.

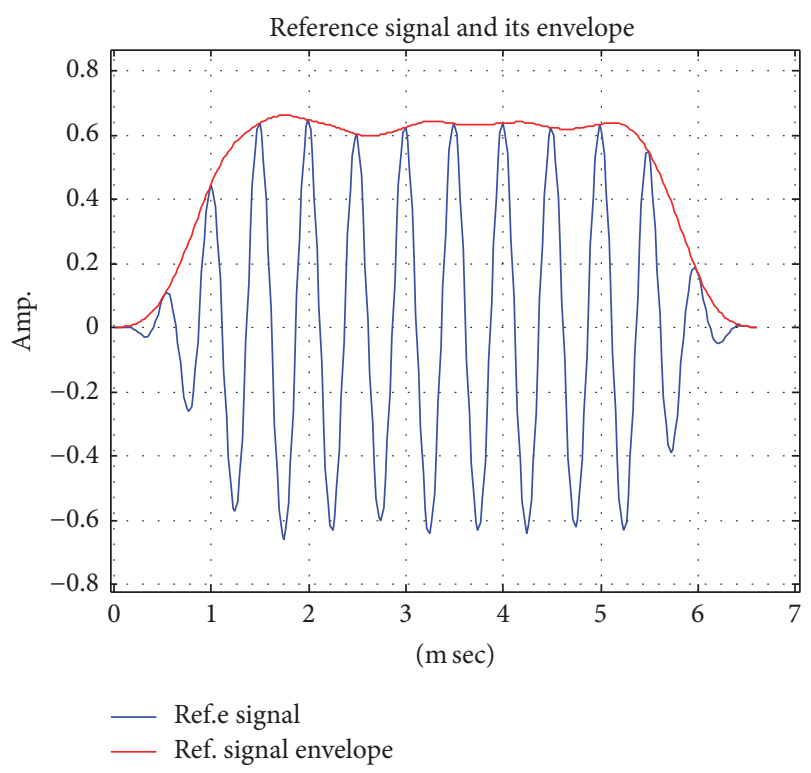

FIGURE 15: Reference signal and its envelope using Hilbert transform.

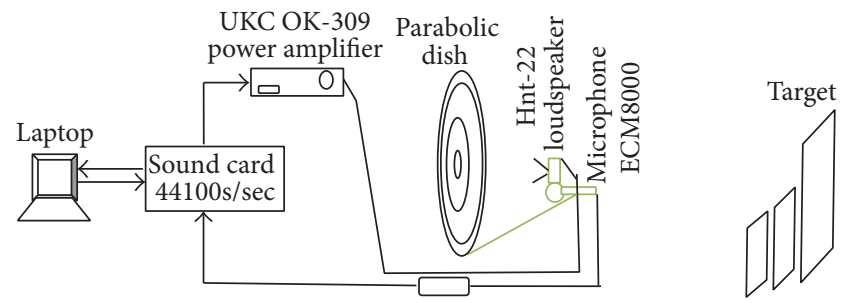

FIGURE 16: Schematic representation of experimental setup used for proposed method verification.

\section{Experimental Examples}

The proposed method is experimentally tested using single, double, and triples targets and a directed acoustic transmission-reception system with a parabolic dish with a focal length of $35 \mathrm{~cm}$. The parabolic dish has an elliptical aperture with a major axis of $65 \mathrm{~cm}$ and a minor axis of $60 \mathrm{~cm}$. Figure 16 shows a schematic representation of experimental setup used for proposed method verification.

In all experiments, signals are sampled at the sampling frequency $f_{\mathrm{s}}=44.1 \mathrm{kHz}$ over an acquisition time $T=1 \mathrm{sec}$, so the number of samples acquired is $N=44100$ samples, discrete Fourier transform (DFT) is computed with a 100000point FFT, and the frequency increment is $d f=1 \mathrm{~Hz}$.

5.1. Single Target. At first, a single board of wood with dimensions $90 \times 60 \mathrm{~cm}$ is used as a target, which is located at about $330 \mathrm{~cm}$ from the directed acoustic system, as shown in Figure 16. The modified transform function $h m(t)$ (red curve) and its absolute value (blue curve) are shown in Figure 17.

From Figure 17, target distance is $d=((19.27 \mathrm{~m} \cdot \mathrm{sec} \times$ $340 \mathrm{~m})) / 2=327.6 \mathrm{~cm}$; the result is satisfactorily close to the real value. 


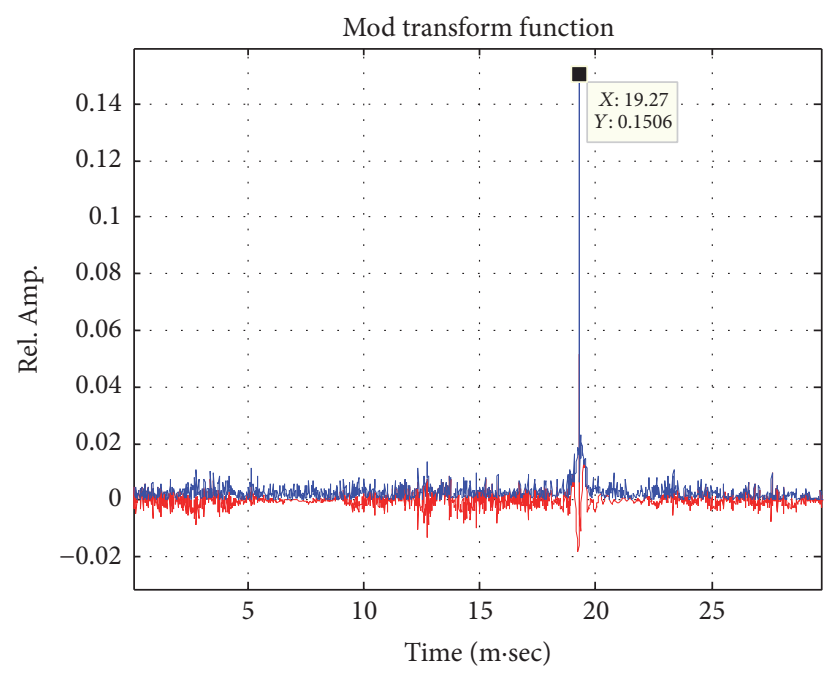

FIGURE 17: Modified transform function $h m(t)$ of single target.

5.2. Double Target. Two boards of wood with dimensions 60 $\times 40 \mathrm{~cm}$ and $90 \times 60 \mathrm{~cm}$ are used as a target (the smaller is in front, and the distance between them is about $30 \mathrm{~cm}$ ); the target is located at about $8 \mathrm{~m}$ from the directed acoustic system, as shown in Figure 16. Figures 18, 19, and 20 show the received signal, the transform function $h(t)$ with $\gamma=0.001$, and the modified transform function $h m(t)$ of third-order iteration, respectively.

From Figure 20, target distance is $d=((46.69 \mathrm{~m} \cdot \mathrm{sec} \times$ $340 \mathrm{~m})) / 2=7.94 \mathrm{~m}$, and distance between two targets is $d_{1,2}$ $=(((48.5-46.69) \mathrm{m} \cdot \mathrm{sec} \times 340 \mathrm{~m})) / 2=30.77 \mathrm{~cm}$; results are satisfactorily close to real values.

5.3. Triple Target. Three boards of wood with dimensions 60 $\times 40 \mathrm{~cm}, 90 \times 60 \mathrm{~cm}$ and $160 \times 140 \mathrm{~cm}$ are used as a target (the smallest board is the closest to the system and the biggest one is the farthest); the target is located at about $9.25 \mathrm{~m}$ from the directed acoustic system, as shown in Figure 16, the distance between the first board and the second one is about $45 \mathrm{~cm}$, and the distance between the second board and the third one is about $80 \mathrm{~cm}$; Figure 21 shows the received signal, Figure 22 shows the transform $h(t)$ with $\gamma=0.006$, and the modified transform function $h m(t)$ of third-order iteration is shown in Figure 23.

From Figure 23, target distance is $d=((54.44 \mathrm{~m} \cdot \mathrm{sec} \times$ $340 \mathrm{~m})) / 2=9.255 \mathrm{~m}$, the distance between first and second target is $d_{1,2}=(((57.19-54.44) \mathrm{m} \cdot \mathrm{sec} \times 340 \mathrm{~m})) / 2=46.75 \mathrm{~cm}$, and the distance between second and third target is $d_{2,3}=$ $(((62.13-57.19) \mathrm{m} \cdot \mathrm{sec} \times 340 \mathrm{~m})) / 2=83.98 \mathrm{~cm}$; results are satisfactorily close to real values.

\section{Conclusion}

This paper presents a method to separate overlapping echoes using a superresolution algorithm based on experimental echo shape modelling.

The simulation results implemented under MATLAB show a good performance of the method at low SNR.

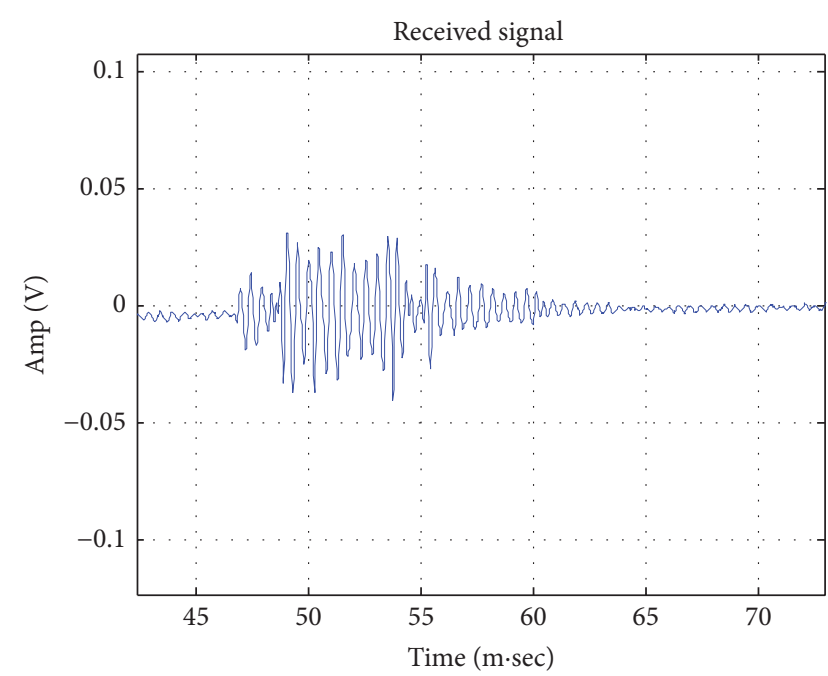

Figure 18: Received signal of double target at $8 \mathrm{~m}$ distance.

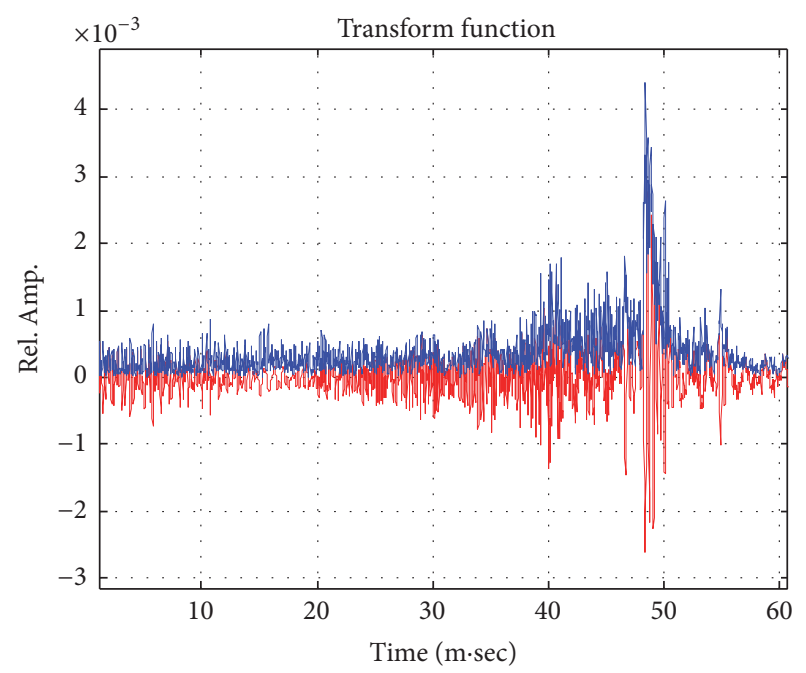

FIGURE 19: Transform function $h(t)$ with $\gamma=0.001$.

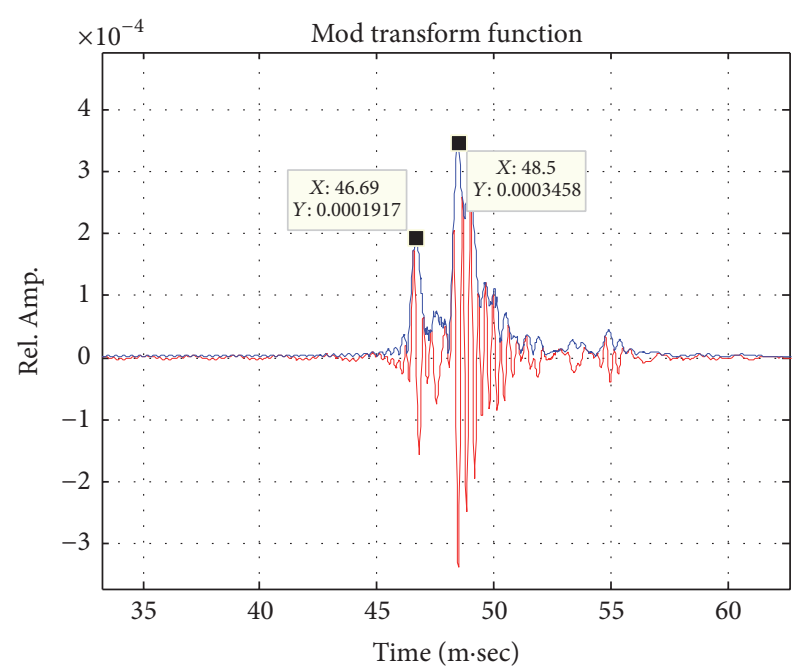

FIGURE 20: Modified transform function $h m(t)$ of third-order iteration with $\gamma=0.001$. 


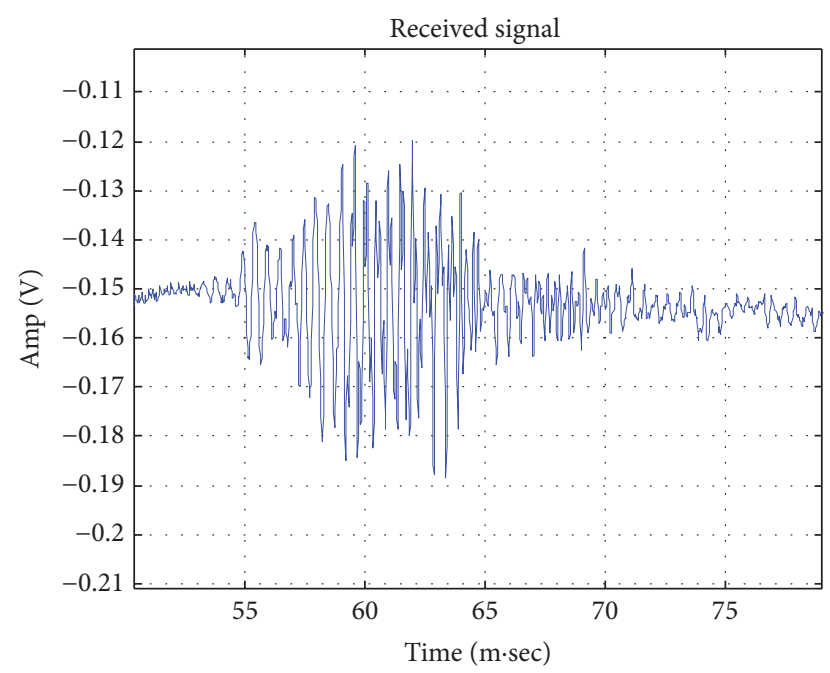

FIGURE 21: Received signal of triple target at $9.25 \mathrm{~m}$ distance.

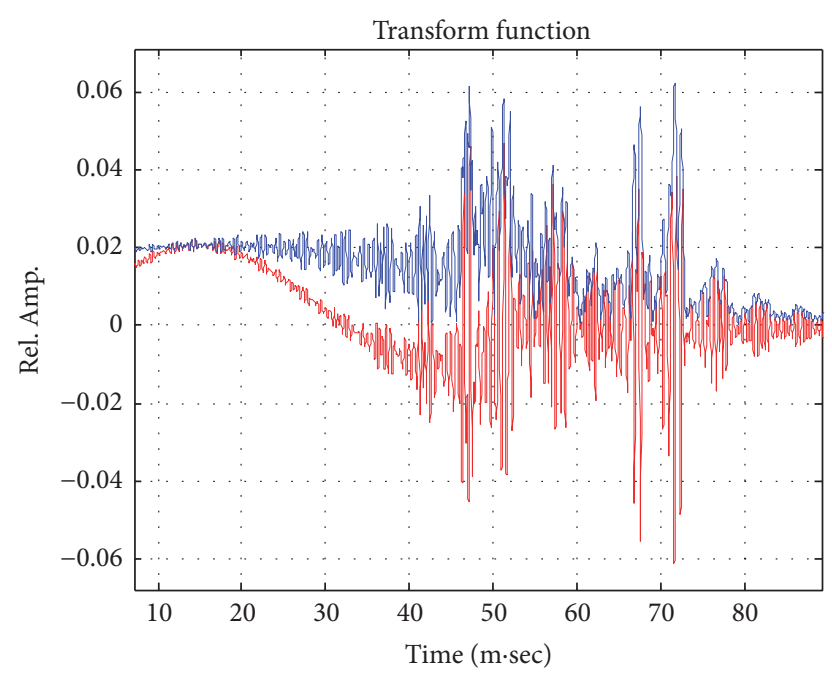

FIgURE 22: Transform function $h(t)$ with $\gamma=0.006$.

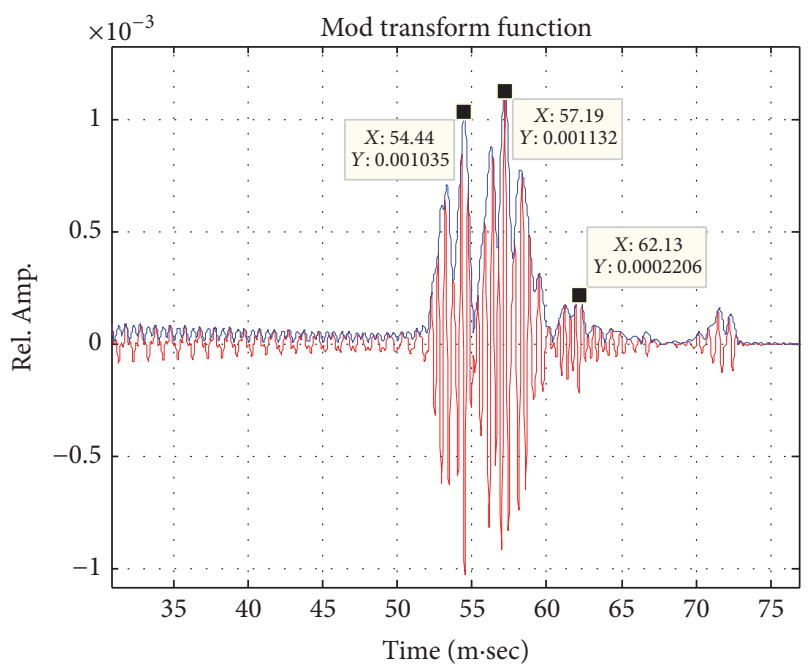

FIGURE 23: Modified transform function $h m(t)$ of third-order iteration with $\gamma=0.006$.
The method is experimentally tested using acoustic transmission-reception system and multiple targets; the method provides accurate localization of multiple targets due to a good accuracy in determining the time delays of individual echoes reflected from each target. Although amplitudes of individual echoes, obtained by this method, are different from the real amplitudes and smaller than them, each echo's amplitude relative to others is respected.

This study adds a new possibility of overlapping echoes separation and can be widely used in a range of applications such as military and biomedical applications.

Developments can be added to improve SNR and determine the amplitude of individual echoes that allow reconstructing the composite signal.

\section{Conflicts of Interest}

The authors declare that there are no conflicts of interest regarding the publication of this paper.

\section{References}

[1] D. M. J. Cowell and S. Freear, "Separation of overlapping linear frequency modulated (LFM) signals using the fractional fourier transform," IEEE Transactions on Ultrasonics, Ferroelectrics, and Frequency Control, vol. 57, no. 10, pp. 2324-2333, 2010.

[2] Y. Lu, A. Kasaeifard, E. Oruklu, and J. Saniie, "Fractional fourier transform for ultrasonic chirplet signal decomposition," Advances in Acoustics and Vibration, vol. 2012, Article ID 480473, 13 pages, 2012.

[3] E. G. Sarabia, J. R. Llata, S. Robla, C. Torre, and J. P. Oria, "Accurate estimation of airborne ultrasonic time-of-flight for overlapping echoes," Sensors (Switzerland), vol. 13, no. 11, pp. 15465-15488, 2013.

[4] R. S., S. C. X., and M. S., "Estimation of signal parameters for multiple target localization," ICTACT Journal on Communication Technology, vol. 5, no. 4, pp. 1039-1044, 2014.

[5] J. Martinsson, F. Hägglund, and J. E. Carlson, "Complete postseparation of overlapping ultrasonic signals by combining hard and soft modeling," Ultrasonics, vol. 48, no. 5, pp. 427-443, 2008.

[6] J. Woodruff, Y. Li, and D. Wang, "Resolving overlapping harmonics for monaural musical sound separation using pitch and common amplitude modulation," in Proceedings of the 9th International Conference on Music Information Retrieval (ISMIR'08), pp. 538-543, USA, September 2008.

[7] K. Reinhold, "Comparison of frequency estimation methods for reflected signals in mobile platforms," World Academy of Science, Engineering and Technology, vol. 57, pp. 147-150, 2009.

[8] S. Zhang, M. Xing, R. Guo, L. Zhang, and Z. Bao, "Interference suppression algorithm for SAR based on time-frequency transform," IEEE Transactions on Geoscience and Remote Sensing, vol. 49, no. 10, pp. 3765-3779, 2011.

[9] R. C. Gonzalez and R. E. Woods, Digital Image Processing, Prentice Hall, 3rd edition, 2008.

[10] J. DiBiase Hector, A high-accuracy, low-latency technique for talker localization in reverberant environments using microphone arrays, Diss. Brown University, 2000.

[11] G. John and G. Proakis Dimitris, Digital Signal Processing. Principles, Algorithms and Applications, Prentice Hall International, 3rd edition, 1996. 
[12] S. Adrián-Martínez, M. Bou-Cabo, I. Felis et al., "Acoustic signal detection through the cross-correlation method in experiments with different signal to noise ratio and reverberation conditions," in Proceedings of the International Conference on Ad-Hoc Networks and Wireless, Springer, Berlin, Germany, 2014.

[13] R. W. Gerchberg, "Super-resolution through error energy reduction," Optica Acta, vol. 21, no. 9, pp. 709-720, 1974.

[14] M. A. Azpúrua, M. Pous, and F. Silva, "Decomposition of Electromagnetic Interferences in the Time-Domain," IEEE Transactions on Electromagnetic Compatibility, vol. 58, no. 2, pp. 385-392, 2016.

[15] K. Dragomiretskiy and D. Zosso, "Variational mode decomposition," IEEE Transactions on Signal Processing, vol. 62, no. 3, pp. 531-544, 2014. 


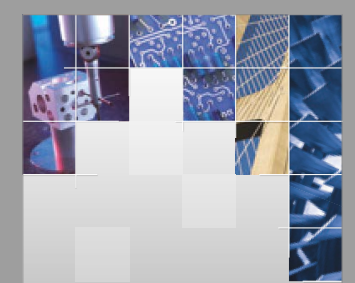

\section{Enfincering}
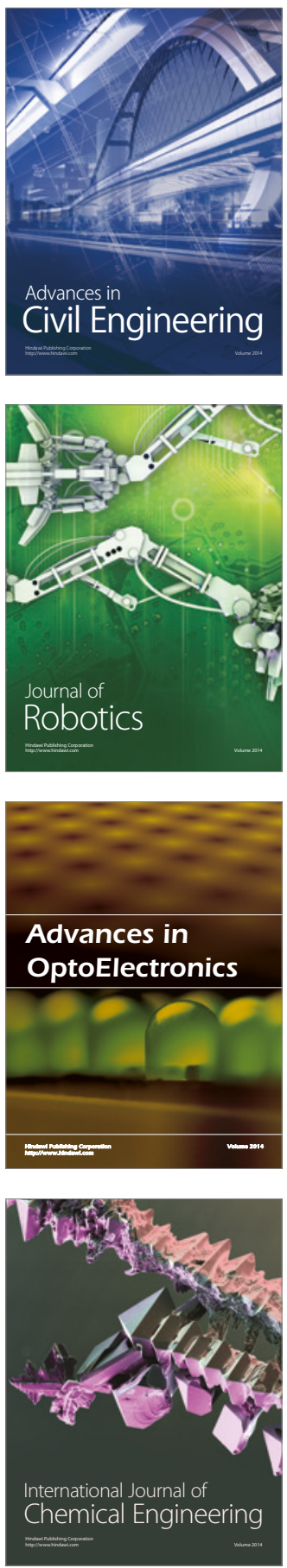

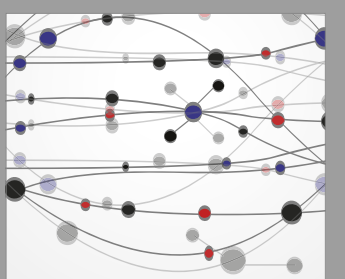

The Scientific World Journal

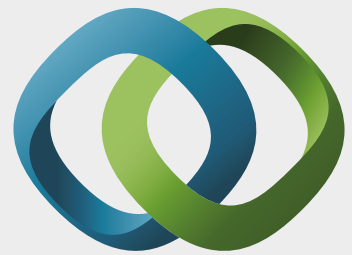

\section{Hindawi}

Submit your manuscripts at

https://www.hindawi.com
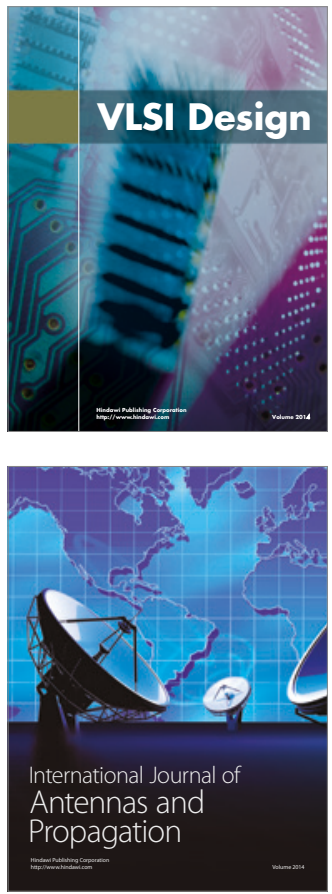

\section{Rotating}

Machinery
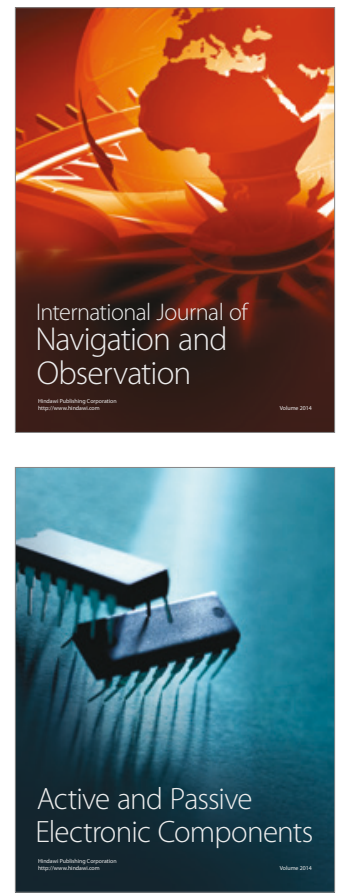
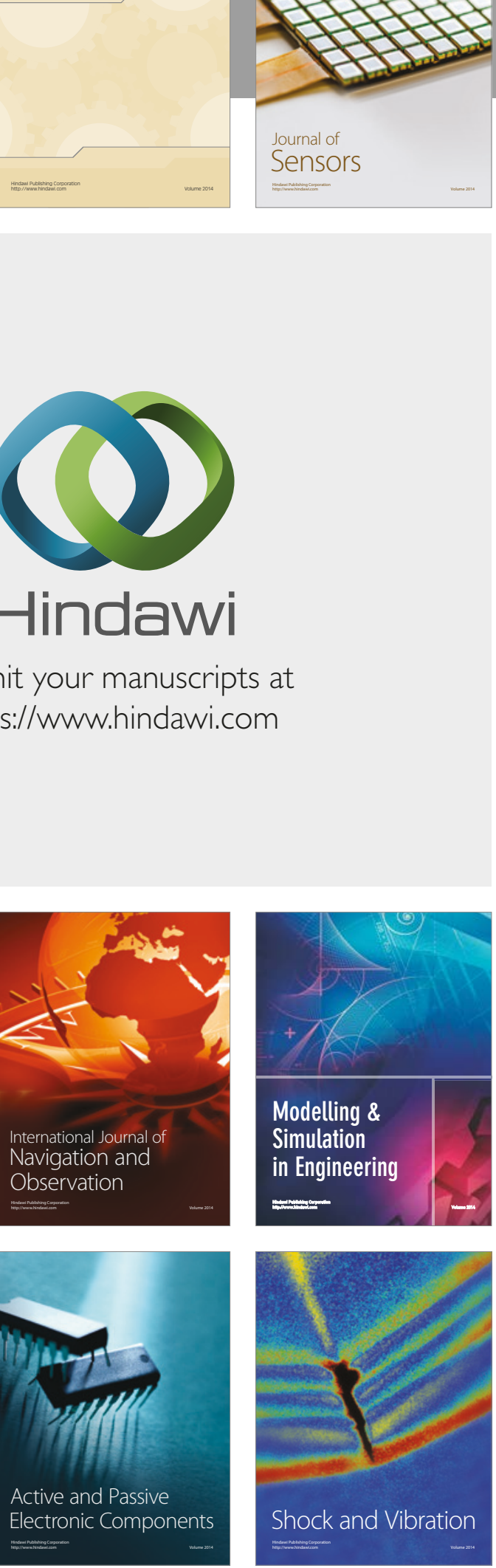
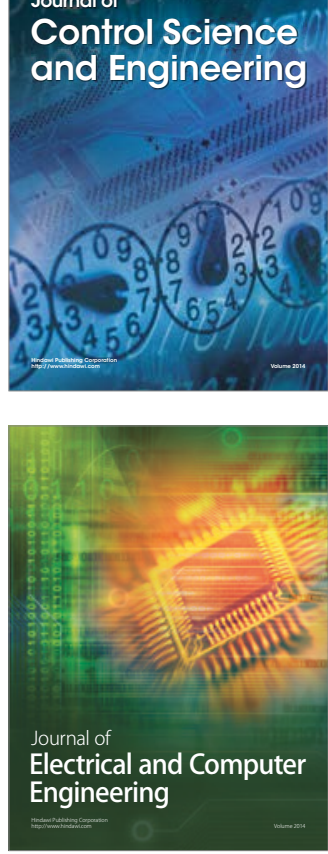

Distributed

Journal of

Control Science

and Engineering
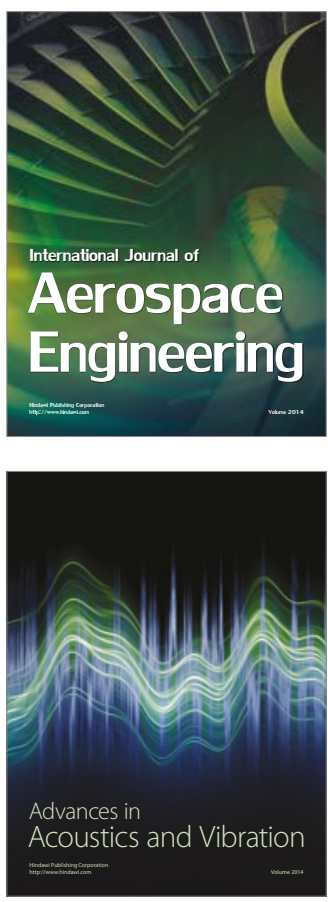

Sensor Networks 